\title{
Desenvolvimento infantil: seus agentes e as políticas públicas do município do Rio de Janeiro
}

\section{| ${ }^{1}$ Elisa Dell'orto Van Eyken, ${ }^{2}$ Carlos Dimas Martins Ribeiro I}

Resumo: O desenvolvimento humano é um processo dinâmico que acontece durante toda a vida. Entretanto, é na infância que eventos externos e fatores internos exercem maior influência. Destacase a desigualdade social entre esses eventos e fatores, porque ela mantém a injusta distância entre as crianças brasileiras vulneradas pela pobreza e as oportunidades que podem mudar essa condição. Essas oportunidades também se relacionam com a família e a possibilidade de cumprir seu papel de agente promotor do desenvolvimento infantil, especialmente o papel da mãe. Este artigo pretende analisar as políticas públicas para a primeira infância do Rio de Janeiro, em 2011, buscando a relação favorável da construção das capacidades básicas das crianças, considerando a condição de agente da mulher que é mãe.

> Palavras-chave: desenvolvimento infantil; relações mãe-filho; políticas públicas.
1 Instituto Federal de

Educação, Ciência e

Tecnologia do Rio de Janeiro;

Programa de Pós-graduação

em Bioética, Ética Aplicada e

Saúde Coletiva; Universidade

Federal Fluminense. Endereço

eletrônico: elisa.eyken@ifrj.

edu.br

${ }^{2}$ Instituto de Saúde da

Comunidade, Universidade

Federal Fluminense. Endereço

eletrônico: dimasmribeiro@

gmail.com

Recebido em: 23/01/2012.

Aprovado em: 21/08/2012 


\section{Introdução}

O Brasil, pelo seu Produto Interno Bruto (PIB), está entre as dez primeiras economias do mundo (IMF, 2011). No entanto, o Índice de Desenvolvimento Humano (IDH) nacional de 0,718 (ONU-PNUD, 2011, p. 134) mantém o Brasil fora dos 50 países mais bem colocados. O coeficiente de Gini de 0,566 revela que $1 \%$ da população brasileira detém uma renda equivalente à renda de $50 \%$ dos brasileiros mais pobres (BARROS et al., 2007; COTA et al., 2007), mantendo uma desigual distribuição de renda. $\mathrm{O}$ Rio de Janeiro, em relação ao desenvolvimento de sua população, está na $58^{\mathrm{a}}$ posição entre as cidades do Brasil, com um IDH de 0,842 e o coeficiente Gini de 0,62 (ONU-PNUD, 2003). Assim, pode-se dizer que pobres são aqueles brasileiros cuja distribuição desigual da renda os afasta de um desenvolvimento que alcance oportunidades.

As oportunidades para se alcançar determinados objetivos, dependem, segundo Amartya Sen (2001), da igualdade de capacitaçôes. Uma vez igualadas, as capacitações representam oportunidades reais para se alcançar objetivos.

Para Sen (2000, p. 10), “desenvolvimento consiste na eliminação de privações de liberdade que limitam as escolhas e as oportunidades das pessoas de exercer ponderadamente sua condição de agente". Essa liberdade, referida como liberdade concreta por Martha Nussbaum (2011), é a combinação das capacitaçôes ou a totalidade de oportunidades que permitem as opções para agir social, econômica e politicamente.

Em relação ao desenvolvimento infantil, a liberdade de oportunidades favorece a condição de agente dos familiares, principalmente da mãe, como seus promotores. O desenvolvimento humano é um processo dinâmico que envolve os aspectos: físico, neurológico e cognitivo e acontece durante todo o ciclo vital. Tem como fator determinante e determinador respostas às necessidades do indivíduo em relação ao meio onde vive. (MOLINARI et al., 2005). Existem períodos mais sensíveis do desenvolvimento, como a infância, nos quais um evento, ou a falta dele, pode ter maior influência (PAPALIA; OLDS; FELDMAN, 2006). A desnutrição na infância pode ser um exemplo dessa relação (LIMA et al., 2010).

Este trabalho visa analisar, de forma teórica, as políticas públicas do município do Rio de Janeiro em vigor em 2011, para melhorar o desenvolvimento das crianças que estão na primeira infância. A análise busca os aspectos favoráveis 
para a promoção das capacidades básicas das crianças, considerando a condição

de agente da mulher que é mãe.

\section{Desenvolvimento infantil}

Para a análise do desenvolvimento infantil, várias teorias são propostas, mas é a relação com o meio no qual a criança vive o interesse deste trabalho. Como processo, o desenvolvimento decorre da interação do ser com seu mundo: sua classe social, seu local de vida e sua época. Os facilitadores dessa interação são os cuidadores e o ambiente. Os primeiros interagem através de seu próprio desenvolvimento: sua educação, suas crenças e seus valores. $\mathrm{O}$ ambiente, através do aspecto físico e das práticas de cuidado e educação da criança, em determinada sociedade (LORDELO, 2002).

Para Bronfenbrenner e Morris (2006), o desenvolvimento existe a partir da relação entre os indivíduos e o contexto. É principalmente na fase inicial do desenvolvimento que a interação ativa, regular e por longo período de tempo da criança com pessoas, objetos e símbolos do seu ambiente, se torna efetiva. Essa interação, chamada de processo proximal, pode ser encontrada, por exemplo, no momento da alimentação ou conforto do bebê, ou nas brincadeiras dos pais com as crianças pequenas. A participação nessas atividades favorece as habilidades, a motivação e o conhecimento para engajamento em atividades similares com outros ou consigo mesmo. A participação das crianças em atividades mais complexas com os pais favorece a formação dessas crianças e as transforma em agentes do próprio desenvolvimento.

Os processos proximais são as engrenagens primárias para o desenvolvimento. Bronfenbrenner propõe que o efeito desses no desenvolvimento varia quando visto em conjunto com o ambiente, no qual o desenvolvimento acontece. $\mathrm{O}$ modelo de investigação do desenvolvimento do autor é conhecido como Processo, Pessoa, Contexto, Tempo (PPCT). Neste modelo, fatores biológicos e genéticos são levados em consideração; estes influenciam a forma, a força, o conteúdo e a direção do processo proximal, assim como o desfecho do desenvolvimento, ao longo do tempo. Por considerar o contexto como uma relação bidirecional, o indivíduo é capaz de modificá-lo e dessa forma interferir na cultura da sua época (BRONFENBRENNER; MORRIS, 2006; BRONFENBRENNER, 1979). 
O que se espera do desenvolvimento é a liberdade para fazer escolhas e a igualdade de oportunidades; somente possíveis pelo desenvolvimento dos funcionamentos, por meio do processo proximal.

\section{Oportunidades}

A pobrezaéconsiderada um risco para o desenvolvimento infantil. Isto se dá porque, ao limitar a quantidade de recursos das famílias, limita o desenvolvimento dos funcionamentos básicos, inatos das crianças. Os funcionamentos ou capacitações básicas são fundamentais para que não haja desvantagem no desenvolvimento de outras capacitaçôes, mais complexas ou combinadas, que permitem as iguais oportunidades na vida dos adultos. Nas palavras de Marta Nussbaum (2011, p. 20) "[as capacitações são] as respostas para a questão, 'o que essa pessoa é capaz de fazer ou de ser?' [...] Um conjunto de (geralmente correlacionadas) oportunidades para ecolher e para agir".

Desta forma, é a desigualdade social que mantém a injusta distância entre as crianças brasileiras vulneradas pela pobreza e as oportunidades que podem mudar essa condição. Porque, mesmo que tenham desenvolvido os funcionamentos básicos, necessários para o desenvolvimento das capacitações, é fundamental a continuidade de estímulos, tais como a saúde, a educação e a socialização. É a combinação das capacitações internas com as condiçôes externas que criam as reais possibilidades de escolha no futuro (NUSSBAUM, 2011). Se não há condição de desenvolvimento das capacitações, que igualdade de oportunidade essas crianças terão quando adultas?

No processo de construção das capacitações, as experiências são consideradas importantes para crianças em desenvolvimento. Quando participam de atividades consideradas práticas culturais, tais como frequentar creches, as crianças se tornam socialmente competentes. Tudge et al. (2006) observaram as atividades diárias de crianças pré-escolares de famílias de classe média, cujos pais tinham curso superior e trabalhavam em suas profissões e famílias cujos pais possuíam mínima escolaridade e eram operários sem qualificação, nos Estados Unidos da América, no Brasil e no Quênia. As crianças foram observadas em seus ambientes diários em relação às atividades relevantes para a escola. Os achados demonstraram as diferenças das sociedades no que diz respeito às expectativas 
relacionadas ao tipo de atividade diária das crianças. Como exemplo, podemos citar a maior preocupação dos pais brasileiros em relação aos cuidados com seus filhos e em relação às atividades de estímulo cognitivo dos pais americanos. Pareceu claro que nas sociedades, quando grupos dominantes criam as regras consideradas apropriadas (creches que tenham atividades de estímulo cognitivo), aqueles que agem de forma diferente provavelmente estão em desvantagem.

Esta desvantagem pode ser vista em relação ao estímulo para o conjunto de funcionamentos das crianças. Ou seja: se colocar uma criança em uma creche que oferece o cuidado e estímulos necessários para o desenvolvimento de habilidades cognitivas é considerado apropriado em determinada sociedade, o fato de não fazer essa escolha afetará a construção das capacitações que tornam as crianças capazes de fazer escolhas, quando adultas. Neste caso, não há diferença entre países ricos e pobres, desenvolvidos ou em desenvolvimento, pois a desigualdade pode estar presente em qualquer contexto.

As oportunidades são liberdades de escolhas e estarão presentes quando houver desenvolvimento no contexto. No caso do desenvolvimento infantil, não podemos esquecer a importância do papel da mulher, que é mãe, de agente promotor dos funcionamentos de seus filhos. Para o exercício desse papel, é necessário que a mulher seja independente e tenha poder de decisão por ser informada e qualificada para isso. Dessa forma, "[...] o papel limitado da condição de agente ativa das mulheres afeta gravemente a vida de todas as pessoas - homens e mulheres, crianças e adultos" (SEN, 2000, p. 222).

Como um dos objetivos para o milênio, o Programa das Nações Unidas para o Desenvolvimento escolheu promover a igualdade entre os sexos e autonomia das mulheres. Na base da desigualdade de gênero, ainda temos os indicadores socioeconômicos e demográficos relacionados com um novo papel social (ou de agente das mudanças sociais) que está sendo assumido pelas mulheres. Como exemplo, podemos considerar a chefia de família (BRASIL, 2002). Além do trabalho fora do domicílio, as mulheres ainda assumem o serviço do lar e o cuidado com os filhos, acumulando em média 22,3 horas por semana nessas tarefas. Essa dupla jornada de trabalho se deve ao fato de que a desigualdade de gênero se relaciona não só com trabalho e renda, mas também com o papel social identificado para o homem e para a mulher (PINHEIRO et al., 2008). 
É justamente nessa relação difícil, entre o papel social identificado para as mulheres (no cuidado com os filhos) e as oportunidades reais para a promoção das suas próprias capacitações, que se encontra o desenvolvimento infantil.

$\mathrm{Na}$ sua condição de agente da mudança social, a mulher deve assumir a responsabilidade de escolha, levando em consideração seu próprio bem-estar. Essa consideração tem sido relacionada com variáveis como: “o potencial [...] para auferir uma renda independente, encontrar emprego fora de casa, ter direito de propriedade, ser alfabetizada e participar como pessoa instruída nas decisões dentro e fora da família” (SEN, 2000, p. 222). No entanto, é importante que o Estado promova acesso igualitário aos meios sociais para seu bem-estar e para facilitar as decisões, inclusive em relação à criação dos filhos. Dessa forma, sem um papel social imposto, fica a liberdade de escolha de acordo com os objetivos individuais de vida (SANTOS, 2007) e a oportunidade da mulher "exercer ponderadamente sua condição de agente" (SEN, 2000, p. 10)

Em relação ao perfil das mães brasileiras, de acordo com os dados da Síntese dos Indicadores Sociais (SIS) de 2010 (BRASIL, 2010), o maior número de mulheres-mães apresentou menos de sete anos de escolaridade. Nesse grupo, 20,3\% tinham entre 15 e 19 anos. No Rio de Janeiro, no ano de 2005, mais de $17 \%$ dos nascidos vivos tiveram mães adolescentes e mais de $29 \%$ das mães adolescentes eram recorrentes. A maior parte da população de mães adolescentes possuía baixa escolaridade e não possuía trabalho fora de casa (SILVA et al., 2011).

$\mathrm{O}$ que tem sido proposto pelas políticas públicas para o desenvolvimento infantil que favoreça a condição das mães, no seu papel de agente?

\section{Políticas públicas para o desenvolvimento infantil no Rio de Janeiro}

A Secretaria de Assuntos Estratégicos do Governo federal, através de seus representantes, tem declarado na mídia seus alvos prioritários para políticas de longo prazo, visando, em particular, à manutenção do poder aquisitivo, conquistado pela nova classe média. Importante salientar que, segundo os dados da própria secretaria, ainda precisamos de 15 anos para chegar ao mesmo ponto de países semelhantes em desenvolvimento, no que diz respeito à distribuição de renda. Na pauta de atuação desse órgão, como um dos alvos, está o desenvolvimento pleno na primeira infância (até 3 anos de idade) ${ }^{1}$. 
Na mesma matéria jornalística, uma referência à unificação de políticas sociais com a finalidade de redução das desigualdades chamou a atenção. Diz respeito à assessoria prestada ao município do Rio de Janeiro para um programa de proteção integral à infância, com inclusão da família, que unifique os programas já existentes. Por enquanto, o município conta com políticas, programas e estratégias implantadas ao longo dos anos e por diversos governos. Em resposta às inúmeras necessidades da população pobre, o Rio de Janeiro e mais cinco municípios brasileiros abriram as portas para implantação de uma estratégia-piloto, em 2009.

A estratégia, denominada "Brasileirinhos e Brasileirinhas Saudáveis: primeiros passos para o desenvolvimento nacional” (BRASIL, 2010a), tem como objetivo garantir a qualidade de vida das crianças, através de estímulos ao desenvolvimento físico, emocional, cognitivo e social. Para isso, foca a diminuição da taxa de mortalidade, a atenção ao pré-natal, ao parto, ao aleitamento materno e ao puerpério, além da atenção aos bebês deficientes. Também engloba o planejamento familiar, a atenção ao uso de substâncias psicoativas, a organização de uma rede que favoreça a formação de cuidadores orientados para o desenvolvimento das funcionalidades e a diminuição das vulnerabilidades relacionadas à violência.

$\mathrm{Na}$ caracterização da população de abrangência da estratégia no Rio de Janeiro, a população infantil menor do que sete anos ou na primeira infância representa cerca de $9 \%$ do total de habitantes (cerca de 500 mil) e se concentra nos bairros de Campo Grande, Guaratiba, Santa Cruz, Paciência, Bangu, Realengo, Senador Camará, Pavuna, Maré, Jacarepaguá e Tijuca. Tendo em vista as perspectivas de envolvimento de diversas áreas relacionadas com o cuidado infantil, o planejamento do município, principalmente para esses bairros, envolve estratégias de saúde, através da Saúde da Família (ESF), vinculada ou compartilhada com a educação, através da atuação nos Espaços de Desenvolvimento Infantil (EDI) e os programas de apoio à escola e à creche. E integração da ESF com a assistência social, através do projeto Primeira Infância Completa (PIC) e do Programa Saúde na Escola (PSE). A articulação entre os centros de referência da assistência social (CRAS) e os Centros Especializados da Assistência Social (CREAS), responsáveis pelo programa de transferência de renda Bolsa Família (PBF), implantado como Programa Bolsa Família Carioca, intensifica a redução da pobreza e o desenvolvimento das famílias. ${ }^{2}$ 
No município do Rio de Janeiro, a preocupação com a primeira infância, além da saúde e das necessidades básicas, supridas pela ESF e pela distribuição da renda, respectivamente, tem na socialização e nas oportunidades de aprendizagem um importante foco. Para esse fim, foi criado o Programa Primeira Infância Completa (PIC), que é fruto da parceria da Secretaria Municipal de Educação com as Secretarias de Assistência Social e de Saúde e Defesa Civil. O programa foi criado para atender crianças entre três meses e três anos e 11 meses que não estão matriculadas em creches.

O PIC teve início a partir de um projeto-piloto em dez creches do município e atualmente acontece em 52 creches, com previsão de expansão para 100 creches até 2012. O objetivo do programa é o desenvolvimento infantil saudável e feliz. As ações acontecem aos sábados, em creches públicas, nas quais as crianças ficam, no período das $8 \mathrm{~h}$ às $16 \mathrm{~h}$, sob o cuidado de professores, profissionais de saúde e auxiliares de creche. Durante o tempo que passam na creche as crianças realizam diversas atividades - lúdicas, de saúde, de socialização e cognitivas - enquanto os pais têm o período livre para suas atividades. Também são oferecidas orientações aos familiares, através de roda de conversa que acontece ao final de cada sábado, durante uma hora, sobre o cuidar das crianças e as relações de afeto com elas (PREFEITURA, 2010a).

A utilização dos espaços da creche pelo PIC está disponível aos beneficiados do PBF através do cartão Família Carioca, cartão lançado pela Prefeitura do Rio de Janeiro em 2010, como uma adequação do Programa Bolsa Família à realidade do município, sendo considerado um complemento do PBF. Esse complemento está vinculado à frequência de $90 \%$ das crianças à escola, à participação de um dos responsáveis às reuniões escolares e incentiva o desempenho escolar com a criação de bônus financeiros semestrais (PREFEITURA, 2010b).

No Rio de Janeiro, as ações voltadas para a primeira infância dedicam especial atenção à educação e ao melhor desempenho das crianças no ensino fundamental, por isso essas ações estão concentradas na Secretaria de Educação. Além do PIC, os Espaços de Desenvolvimento Infantil (EDI) funcionam desde outubro de 2009, com o objetivo de atender às necessidades das crianças de três meses a cinco anos e meio de idade de forma integral. Os EDI se iniciaram com uma primeira unidade em Bangu, tendo se expandido para 18 unidades. Os espaços atendem a crianças de forma integral. 
Em relação à saúde, o PSE, com a presença de um técnico de enfermagem e a visita semanal de uma unidade móvel, tem como objetivo identificar problemas de saúde, tais como auditivo, ocular e bucal, e encaminhar para as Unidades de Saúde da Família. Além do Programa Saúde na Escola, as ações em saúde ainda englobam alimentação saudável, atividades físicas e exposição adequada ao sol. O desenvolvimento cognitivo e social se deu através do manuseio e leitura de livros e outras atividades de estimulação motora e intelectual (manuseio de quebra-cabeça, massinha, etc.) e também brincadeiras livres e momentos de descanso. Através da vivência de rotinas de acordo com a sua faixa etária, a criança será estimulada na construção das suas capacidades. O espaço também tem por objetivo, pela possibilidade de flexibilidade de horário, das 7 h15min até as $17 \mathrm{~h} 15 \mathrm{~min}$, facilitar a adequação dos horários das famílias com os horários das crianças (PREFEITURA, 2010a).

\section{Discussão}

Nas estratégias do município do Rio de Janeiro para a primeira infância, estão a criança e seu desenvolvimento com saúde, voltado para o melhor desempenho cognitivo e social, com vistas ao melhor aproveitamento na educação fundamental. Estes são, sem dúvida, ingredientes facilitadores das capacidades básicas necessárias para a formação de pessoas com iguais oportunidades.

Em relação aos agentes familiares da promoção do desenvolvimento infantil, estão disponíveis orientações quanto aos cuidados de higiene e de alimentação e de estimulação cognitiva e afetiva. Também está assegurado o tempo livre para a execução de outras atividades, como trabalho, estudo e lazer. As políticas e programas nacionais de atenção à criança, como os presentes na cidade do Rio de Janeiro, tiveram como base programas internacionais como o chileno Chile Crece Contigo e programas nacionais, como o Primeira Infância Melhor (PIM).

Implementado no ano de 2006, Chile Crece Contigo, "representa para o país uma inovação em matéria de políticas públicas de proteção social”. (VILLALOBOS; MILMAN, 2010, p. 26). É parte de uma série de políticas de proteção para famílias pobres e vulneráveis e tem como foco o bem-estar e a segurança das crianças, desde o nascimento até a entrada na escola. O programa também realiza suporte para as famílias e comunidades e especialmente para mães que trabalham. Dessa forma, tenta assegurar os direitos e as oportunidades de desenvolvimento das crianças. 
Outra base para as ações do Rio de Janeiro, o programa Primeira Infância Melhor (PIM) foi implantado no ano de 2003 no Estado do Rio Grande do Sul (SCHNEIDER; RAMIRES, 2007), estruturado em três eixos: família, comunidade e intersetorialidade. A família, como unidade afetiva, educadora, cuidadora e protetora, é considerada como "um agente competente, ativo e coparticipativo no Programa" (p. 53). Os agentes do programa orientam essa unidade em relação ao desenvolvimento infantil, levando em consideração sua identidade cultural. A comunidade, segundo eixo, é vista como apoio para mobilizar e divulgar ações educativas e de saúde para o desenvolvimento da primeira infância. Por último, a intersetorialidade no atendimento às crianças parte do pressuposto de que ações isoladas ou justapostas da saúde, da educação, da cultura, da assistência social e da justiça apresentam resultados desfavoráveis para as necessidades da população.

Sabendo disso, a articulação dos diferentes setores e níveis do governo, em prol da criança e sua família, forma um modelo integrado para a execução favorável de uma política pública para a infância. Os benefícios em relação ao desenvolvimento infantil na área da Assistência Social estão focalizados na atenção à família e seus integrantes com ações de prevenção e de vigilância social. Essas ações favorecem a socialização e proteção, além da autonomia, e são desenvolvidas pelos Centros de Referência da Assistência Social (CRAS). Estes devem priorizar as famílias do Programa Bolsa Família (PBF), programa de transferência de renda a famílias em situação de vulnerabilidade social, financiado pelo Governo federal. Para o desenvolvimento do PIM, a criança e sua família são vistas como uma unidade articulada às ações governamentais.

Os três programas - "Brasileirinhos e Brasileirinhas Saudáveis: primeiros passos para o desenvolvimento nacional" (e seus programas no Rio de Janeiro), "Chile Crece Contigo" e "Primeira Infância Melhor" -, além da articulação para a criação de espaços complementares às creches para o desenvolvimento infantil, estão em sintonia na relação de importância da atenção integral à criança. Em relação à atenção integral, nos três programas a relação social da criança tem o objetivo de permitir o desenvolvimento de capacidades necessárias para a realização dos seus projetos de vida. Sob a ótica da Bioética da Proteção (SCHRAMM, 2008), essas políticas visam aos vulnerados, ou seja, aqueles que por conta própria não conseguem modificar sua condição, e dão a estes os meios necessários para alcançar seus objetivos de vida. 
No que diz respeito à condição da mulher como agente do desenvolvimento infantil, podem-se considerar de duas formas: a primeira diz respeito à capacitação das mesmas em relação ao cuidado integral dos seus filhos, que está presente nas ações apresentadas. A segunda diz respeito às oportunidades de mudança na sua condição de mulher como pessoa. Para isso, é preciso levar em consideração o bem-estar e o objetivo de vida da mulher. Neste sentido, a não ser pelo tempo livre do cuidado com os seus filhos, é que estas políticas e programas precisam olhar a mulher e não apenas a mãe. Esse olhar está previsto nas ações do CRAS.

Os Centros de Referência de Assistência Social têm na proteção social, além da prevenção dos riscos, a função de fortalecer vínculos familiares e sociais, sobretudo para aqueles em situação de vulnerabilidade social por pobreza e/ ou com vínculos afetivos frágeis. É importante visar ao desenvolvimento da criança, mas também a família deve ser estimulada ao desenvolvimento social e pessoal. A vantagem do trabalho do CRAS é a possibilidade do olhar e do cuidar interdisciplinar, tão importantes para que o indivíduo seja visto como ator de vários cenários. O Programa de Atenção Integral à Família (PAIF), desenvolvido pelo CRAS, tem como um de seus objetivos fortalecer a autonomia dos membros da família, através de aquisições sociais e materiais. Dentre as ações específicas do PAIF, estão o encaminhamento e acompanhamento para benefícios e serviços socioassistenciais ou outras políticas setoriais (BRASIL, 2009).

Uma vez que ações de capacitação estejam disponíveis para a mulher, principalmente para as adolescentes, é possível que as mães (tendo sua condição como pessoa respeitada e fortalecida) também deem mais importância ao bemestar dos seus filhos e melhorem sua condição de agente do desenvolvimento.

Para a formulação de uma política na qual a mulher possa construir suas capacitaçôes, além das maternas, sugere-se pensar os espaços infantis também como espaços de educação e formação para adolescentes e adultos jovens. Isto se dá porque a realidade da mãe atual indica caminhos para políticas que a englobem como mulher.

\section{Conclusão}

O movimento atual do governo do município do Rio de Janeiro, em relação às políticas de desenvolvimento e de manutenção de renda da população carente, está na direção do desenvolvimento infantil. $\mathrm{O}$ caminho parece estar sendo 
traçado, observando o contexto de desenvolvimento das crianças em situação de vulnerabilidade com o objetivo de modificar as condições que possam limitar suas oportunidades, quando adultos.

Apesar do avanço dessas políticas em relação à família e à comunidade na qual a criança está inserida, ainda é pequeno o espaço destinado à liberdade da mulher que é mãe. Como este movimento ainda pode estabelecer modificações importantes na relação da família com a sociedade, é necessário estabelecer políticas efetivas que permitam a liberdade (com responsabilidade) de escolha da mulher, até mesmo em relação à escolha de ser mãe. E, uma vez decidida pela maternidade, que ela também possa optar pelas melhores ações positivas para o desenvolvimento de seus filhos. ${ }^{3}$

\section{Referências}

BARROS, R.P. et al. A queda recente da desigualdade no brasil. Rio de Janeiro: IPEA, 2007. BRASIL. Ministério da Saúde. Secretaria de Atenção à Saúde. Departamento de Ações Programáticas e Estratégicas em Saúde. O futuro hoje: estratégia brasileirinhas e brasileirinhos saudáveis : primeiros passos para o desenvolvimento nacional. V. IV. Brasília : Ministério da Saúde, 2010 (a).

BRASIL. Ministério do Desenvolvimento Social e Combate à Fome. Tipificação nacional de serviços socioassistenciais. Resolução n. 109, de 11 de novembro de 2009. Diário Oficial da União, Brasília, v. 146, Seção 1, n. 255, p. 1-43, 25 nov 2009.

BRASIL. Ministério do Planejamento, Orçamento e Gestão. Instituto Brasileiro de Geografia e Estatística. Diretoria de Pesquisas. Perfil das Mulheres responsáveis pelos domicílios no Brasil 2000. Rio de Janeiro: IBGE, 2002.

BRASIL. Ministério do Planejamento, Orçamento e Gestão. IBGE. Síntese dos indicadores sociais: uma análise das condições de vida da população brasileira. Rio de Janeiro: IBGE, 2010(b).

BRONFENBRENNER, U.; MORRIS, P.A. The bioecological model of human development. In: DAMON, W.; LERNER, R.M. Handbook of child psychology: Theoretical Models of Human Development. V.I. New Jersey: John Wiley \& Sons, 2006.

BRONFENBRENNER, U. The ecology of human development: experiments by nature and design. USA: Harvard University Press, 1979. 330 p.

COTA, R.M.M. et al. Pobreza, injustiça, e desigualdade social: repensando a formação de Profissionais de Saúde. Revista Brasileira de Educação Médica. Rio de Janeiro, v. 31, n. 3, p. 278-286, 2007. 
INTERNATIONAL MONETARY FUND. World Economic and Finacial Sorveys. Slowing Growth, Rising Risks. World Economic Outlook. Washington, DC: International Monetary Fun, September 2011. Disponível em: <http://www.imf.org/external/pubs/ft/ weo/2011/02/pdf/>. Acesso em: 30 nov. 2011.

LIMA, A.L.L. et al. Causas do declínio da desnutrição no nordeste do Brasil (1986-19962006). Revista de Saúde Pública. São Paulo, v. 44, n. 1, p. 17-27, 2010.

LORDELO, E.R.Contexto e desenvolvimento humano: quadro conceitual. In: LORDELO, E.R.; CARVALHO, A.M.A.; KOLLER, S.H. (Orgs.). Infância brasileira e contextos de desenvolvimento. São Paulo: Casa do Psicólogo, 2002. p. 5-18.

MOLINARI, J.S.O.; SILVA, M.F.M.C.; CREPALDI, M.A. Saúde e desenvolvimento da criança: a família, os fatores de risco e as ações na atenção básica. Psicologia Argumento, Curitiba, v. 23, n. 43, p. 17-26, out-dez 2005.

NUSSBAUM, M.C. Creating Capabilities: the human development approach. Cambridge: Harvard University Press, 2011. 237p.

ORGANIZAÇÃO DAS NAÇÕES UNIDAS. Programa das Nações Unidas para o Desenvolvimento. Relatório do Desenvolvimento Humano 2011. Sustentabilidade e Equidade: um futuro melhor para todos. Nova York: PNUD, 2011.

ORGANIZAÇÃO DAS NAÇÕES UNIDAS. Programa das Nações Unidas para o Desenvolvimento. Atlas do Desenvolvimento Humano no Brasil, 2003. Disponível em <http://www.pnud.org.br/atlas/instalacao/index.php.> . Acesso em: 30 out 2011.

PAPALIA, D.E.; OLDS, S.W.; FELDMAN, R.D. Desenvolvimento Humano. Porto Alegre: Artmed, 2006. 888 p.

PINHEIRO, L. et al. Retratos da desigualdade de genenero e raça. Brasília: SPM, 2008. 36p. PREFEITURA DA CIDADE DO RIO DE JANEIRO. Secretaria Municipal de Educação Gerencia Especial de Educação Infantil. Espaço de Desenvolvimento Infantil EDI: modelo conceitual e estrutura. Rio de Janeiro: SME-RJ, 2010a.

PREFEITURA DO RIO DE JANEIRO. Decreto no 32.887, de 8 de outubro de 2010. Diário Oficial do Rio de Janeiro, Rio de Janeiro, v. 24, n.140, p. 3-4, 13 out 2010 (b).

PREFEITURA DO RIO DE JANEIRO. Secretaria Municipal de Educação. Primeira Infância Completa (PIC). Disponível em: <http://www.rio.rj.gov.br/web/sme/ exibeconteudo?article-id=131779> Acesso em: 15 out 2011.

SANTOS, J.A. Igualdade de gênero em alcance: considerações para o estabelecimento de novas relações sociais. Revista Eletrônica de Ciências Sociais. Juiz de Fora, v. I, n. I, p.113-129, fev. 2007. SCHNEIDER, A.; RAMIRES, V.R. Primeira Infância Melhor: uma inovação em política pública. Brasília: Unesco, 2007. 128 p. 
SCHRAMM, F.R. Bioética da Proteção: ferramenta válida para enfrentar problemas morais na era da globalização. Revista Bioética. Brasília, v. 16, n. 1, p. 11-24, 2008.

SEN, A. Desenvolvimento como liberdade. São Paulo: Cia das Letras, 2000. 409 p. . Desigualdade reexaminada. Rio de Janeiro: Record, 2001. 301 p.

SILVA, K.S. et al. Gravidez eecorrente na adolescencia e vulnerabilidade social no Rio de Janeiro (RJ, Brasil): uma análise de dados do Sistema de Nascidos Vivos. Ciência e Saúde Coletiva. Rio de Janeiro, v. 16, n. 5, p. 2485-2493, 2011.

TUDGE, J.R.H. et al. A window into different cultural worlds: yong children's everyday activities in the United States, Brazil, and Kenya. Child Development. Chicago, v. 77, n. 5, p. 1446-1469, Sept-Oct 2006.

VILLALOBOS, V.S.; MILMAN, H.M. Cuatro años creciendo juntos: memoria de la Instalación del Sistema de Protección Integral a la Infancia Chile Crece Contigo 2006-2010. Chile: Gráfica Puerto Madero, 2010.

\section{Notas}

${ }^{1}$ Notícia disponível em: http://www.sae.gov.br/site/, veiculada pelo jornal O Globo, de 1/05/2011, p. 40 .

2 "Brasileirinhos Saudáveis se desenvolvendo no Rio de Janeiro: por uma política de cuidar e de brincar na cidade das crianças maravilhosas”. Estratégia Brasileirinhas e Brasileirinhos Saudáveis. Disponível em: http://www.estrategiabrasileirinhos.com.br/?page_id=246. Acesso: 28 set 2011.

${ }^{3}$ E.D. Van Eyken trabalhou na revisão bibliográfica, na concepção teórica e na confecção do texto. C.D.M. Ribeiro trabalhou na concepção teórica e redação final do texto. 


\section{Abstract}

Child development: its agents and the public policies of the city of Rio de Janeiro

Human development is a dynamic process that occurs throughout life. However, it is during the childhood when external events and internal factors have higher influence. The social inequality stands out among the factors and events because it keeps the unfair distance between the Brazilian children that are vulnerable as a result of the poverty and the opportunities with the ability to change that condition. These opportunities also are related to their families and the possibility to fulfill the role as a promoter of children development, particularly the mother's role. This paper analyzes public policies for early childhood in the city of Rio de Janeiro, in 2011, seeking a favorable relation of building up the children basic abilities, considering the agent status of the woman as a mother.

> Key words: child development; mother-child relation; public policies. 